\title{
ROBUST POSITION CONTROL OF ELECTRO HYDROSTATIC ACTUATOR WITH SYSTEM UNCERTAINTIES USING SLIDING MODE CONTROL AND NEURAL NETWORKS ALGORITHM
}

\author{
$\underline{\text { Kyoung Taik Park }}^{1}$ and Han Me Kim ${ }^{2} *$ \\ ${ }^{1}$ Nano Fusion Production System Research Division, KIMM, Daejeon, Korea \\ ${ }^{2}$ Environmental and Energy Systems Research Division, KIMM, Daejeon, Korea \\ *Corresponding author (kimhanme@gmail.com)
}

\begin{abstract}
This paper deals with robust position control of electro-hydrostatic actuator (EHA) systems with system uncertainties. EHA systems compared with conventional hydraulic actuator (CHA) systems are controlled by electric motor directly connected to bidirectional pump and its size is much smaller due to the component integration such as a pump, a reservoir, various valves and horses. In addition, EHA systems have fast response property because of high stiffness. Therefore, EHA systems can replace CHA systems as well as satisfy system performance. In spite of the merit of EHA systems, EHA systems in construction field are exposed to many system uncertainties due to the distinct characteristics of construction field. However, it is difficult to measure or estimate system uncertainties. Thus, sliding mode controller (SMC) and radial basis function neural networks (RBFNNs) algorithm are designed to compensate system uncertainties and to achieve robust control performance with EHA systems.
\end{abstract}

Keywords: Electro-hydraulic Actuator, System Uncertainty, Sliding Mode Control, Radial Basis Function Neural Networks

\section{INTRODUCTION}

Recently, many construction machineries have been replaced to automation systems in order to improve operation efficiency in construction field. The main automation part of construction machinery is hydraulic actuator part of which basic components are electric motor or diesel engine for generating power, pump, reservoir, various valves, hoses and manifold. Therefore, conventional hydraulic actuator (CHA) systems consisted of many components is bulk and it is difficult to fulfill system maintenance because of operating many hydraulic actuator through one or more manifold and many valves. Especially, since the leakage of working fluid occurs between joints of pump, fluid conduit, manifold and fluid conduit due to the high pressure of working fluid, there is environmental pollution problem. To solve problems of the CHA systems, the CHA part in many construction machineries is necessity to improve about aspects of its size, system maintenance, and environmental pollution.
The electro hydrostatic actuator (EHA) systems have been developed to replace the role of the CHA systems for the realization of a fly-by-wire or a power-by-wire technology for aircraft flight control systems [1]. The EHA systems compared with the CHA systems are very small size hydrostatic actuator system and its independent operation ability make it easy to supervise the operating condition of the hydraulic actuators in aircraft flight systems. In addition, the EHA systems have not only highly energy-efficiency due to high stiffness but also low leakage property of working fluid because of very short connection between components [2]. The operating mechanism of the EHA systems is as follows: the position change of the EHA systems can be executed through the rotation of pump directly connected to electric motor and the velocity change of the EHA systems can be decided by the revolutions per minute (rpm) change of the pump. Consequently, the electric motor of the EHA systems replaces the role of valves in the conventional hydraulic actuator. 
To improve the control performance of construction machinery equipped the EHA systems, robust control scheme should be considered to the control system design because the control systems are exposed to a rough work environment. As robust linear control schemes, there is LQG/LTR(Linear Quadratic Gaussian with Loop Transfer Recovery) and $\mathrm{H}_{\infty}$, representatively[3]. As robust nonlinear control scheme, there is back-stepping, feedback linearization, and sliding mode control schemes, representatively [4, 5]. The LQG/LTR and $\mathrm{H}_{\infty}$ control scheme require a high level of mathematical technique and are very complex. In the case of back-stepping and feedback linearization control schemes, there is necessity to know information for the nonlinear term. On the other hand, the sliding mode control scheme consisted of equivalent term for linear system and robust term for unknown system uncertainty does not require exact information for system uncertainties when compared with above mentioned control schemes. However, even if system uncertainties are compensated by the sliding mode control scheme, there is the limitation of control performance because the sliding mode control scheme has bounded information for the system uncertainties.

In this paper, to give robust property and position tracking for the EHA systems with the system uncertainties, the sliding mode control scheme based on the EHA system dynamics is designed. In addition, to compensate the unknown uncertainties of the EHA systems, RBFNNs(Radial Basis Function Neural Networks) algorithm as auxiliary compensator is designed.

In section 2, the EHA systems are mathematically modeled. In section 3, the SMC scheme as the main controller and the RBFNNs algorithm as the auxiliary compensator are considered for the EHA systems. In section 4, computer simulation is executed for evaluating the tracking performance and robustness of the proposed control system. Finally, the conclusion of the paper is given in section 6.

\section{SYSTEM MODELING}

Figure 1 shows the simplified schematic diagram of an EHA system that consists of an electric servo motor, bi- directional gear pump and actuator. The servo motor rotates the gear pump and then generates the flow rate. The pressure generated by the flow rate changes the position of the piston rod. The movement direction of the piston is related to the rotational direction of the servo motor. The chamber volumes of the actuator depend on the cross sectional area and the displacement of the piston as follows:

$\left\{\begin{array}{l}V_{A}(t)=V_{0 A}+A x(t) \\ V_{B}(t)=V_{0 B}-A x(t)\end{array}\right.$

where $V$ and $V_{0}$ are the chamber volume and the initial chamber volume, respectively, $A$ and $x$ are the pressure area of a double rod hydraulic cylinder and displacement of the piston, respectively, and subscripts ' $A$ ' and ' $\mathrm{B}$ ' denote the chamber notations of the actuator.

Considering the fluid compressibility and continuity principle for the actuator, the flow rate equations of both ports of the actuator can be represented as [6]

$\left\{\begin{array}{l}Q_{A}=A \dot{x}+\frac{V_{0 A}+A x}{\beta_{e}} \dot{P}_{A}+L P_{A} \\ Q_{B}=A \dot{x}-\frac{V_{0 B}-A x}{\beta_{e}} \dot{P}_{B}-L P_{B}\end{array}\right.$

where $Q$ is the flow rate in the actuator, $\beta_{e}$ is the effective bulk modulus of the working fluid, and $L$ and $P$ are the actuator external leakage coefficient and the pressure in the chamber, respectively.

We assume that there is no fluid leakage of conduits because the conduits of EHA systems are very short and hard. Then, Eq. (2) can be expressed as

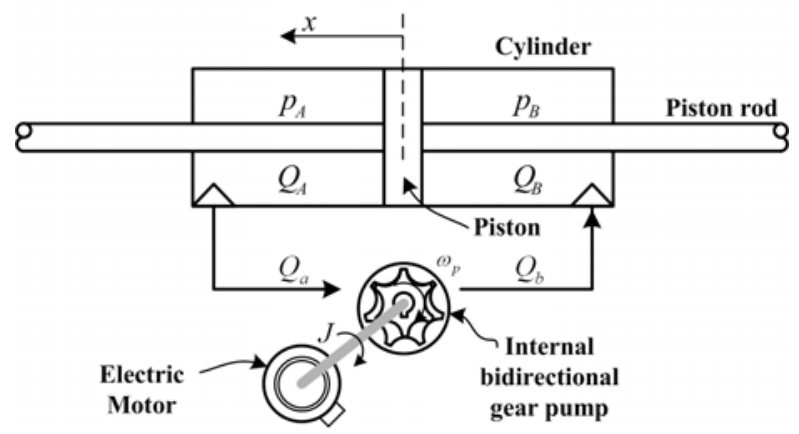

Fig. 1 Simplified schematic diagram of an EHA system 
$\left\{\begin{array}{l}Q_{A}=A \dot{x}+\frac{V_{0 A}+A x}{\beta_{e}} \dot{P}_{A} \\ Q_{B}=A \dot{x}-\frac{V_{0 B}-A x}{\beta_{e}} \dot{P}_{B} .\end{array}\right.$

The electric motor, directly connected to the hydraulic pump, changes the flow direction and adjusts the flow rate through the ports. In addition, the pressure generated by the continuous supply of flow in the actuator may produce a minute fluid leakage of the pump. Hence, the equations for the fluid leakage of the pump are expressed as

$$
\left\{\begin{array}{l}
Q_{a}=C_{p} \omega_{p}-L_{f} P_{L} \\
Q_{b}=-Q_{a}
\end{array}\right.
$$

where $Q$ is the flow rate of the pump, whose subscripts ' $a$ ' and ' $b$ ' denote the ports of the pump, $C_{p}$ is the volumetric capacity of the pump, $\omega_{p}$ is the rotational velocity of the electric motor, $L_{f}$ is the leakage factor of the pump and the load pressure $P_{L}=P_{A}-P_{B}$. From Eq. (4), the inflow and outflow of the pump are expressed as function of the rotational velocity $\omega_{p}$.

The actuator dynamic equation of EHA systems is expressed as

$\left(P_{A}-P_{B}\right) A=M \ddot{x}+F_{e x}$

where $M$ and $x$ are the mass and displacement of the piston, respectively, and $F_{e x}$ is the external disturbance force.

In order to substitute Eq. (3) into Eq. (5), the derivative of Eq. (5) is expressed as

$\left(\dot{P}_{A}-\dot{P}_{B}\right) A=M \dddot{x}+\dot{F}_{e x}$.

From Eq. (6), we assume that the conduits connected between the actuator ports and the pump ports are very short. Then, the flow rates in Eqs. (3) and (4) can be represented as $Q_{A}=Q_{a}$ and $Q_{B}=Q_{b}$. Substituting Eqs.

(1) through (4) into Eq. (6), the dynamic equation of EHA systems can be represented as

$$
\begin{aligned}
\dddot{x}= & -\frac{1}{M}\left\{\beta_{e} A^{2}\left(\frac{1}{V_{A}}+\frac{1}{V_{B}}\right) \dot{x}+\dot{F}_{e x}\right\} \\
& +\frac{\beta_{e} A}{M}\left(\frac{1}{V_{A}}+\frac{1}{V_{B}}\right)\left(L_{f} P_{L}-C_{p} \omega_{p}\right) .
\end{aligned}
$$

\section{CONTROLLER DESIGN}

\section{A. Sliding Mode Control}

To design a SMC system, Eq. (7) can be transformed to a general form as follows[7]:

$\dddot{x}=\Phi \dot{x}+B u+\xi$

where

$\Phi=-\frac{1}{M}\left(\frac{1}{V_{A}}+\frac{1}{V_{B}}\right) \beta_{e} A^{2}, \quad B=\frac{\beta_{e} A\left(V_{A}+V_{B}\right) C_{p}}{M V_{A} V_{B}}$,

$\xi=-\frac{1}{M} f, u=\omega_{p}$.

To trap a state to the line called to the sliding surface, the sliding surface is defined as

$s(t)=\ddot{e}(t)+2 \lambda \dot{e}(t)+k_{i} \int_{0}^{t} e(\tau) d \tau+\lambda^{2}$

where $\lambda$ and $k_{i}$ are a positive design parameters. $e=x_{d}-x$ in which $x_{d}$ is a desired position trajectory.

If the sliding surface is reached and an ideal sliding motion takes place in arbitrarily finite time, the sliding surface satisfies $s(t)=0$ which in turn implies that $\dot{s}(t)=0$. Therefore, the equivalent control law at a low frequency can be obtained as follows:

$u_{e q}=\frac{1}{b}\left(\dddot{x}_{d}+2 \lambda \ddot{e}+\lambda^{2} \dot{e}+k_{i} e\right)$.

To derive the SMC with the robust term for a high frequency range, the Lyapunov candidate function is chosen as a quadratic form, as follows:

$V(t)=\frac{1}{2} s^{2}>0$

The time derivative of Eq. (14) can be represented as follows:

$\dot{V}(t)=s(t) \dot{s}(t)<0, \quad s(t) \neq 0$.

From Eq. (15), if the time derivative is a negative definite function, the control system has stability and the states of the system converge to the sliding surface.

The sliding mode control for the low and the high frequency components is defined as follows:

$u=u_{e q}+u_{r b}$

where $u_{r b}$ is the robust term of the SMC.

Substituting Eq. (16) into Eq. (15), the robust control term satisfying the reaching condition of Eq. (15) can be obtained such that

$u_{r b}=k_{r b} \operatorname{sgn}(s(t))$

where $k_{r b}$ is a positive design parameter. The signum function $\operatorname{sgn}(\cdot)$ is defined as 
$\operatorname{sgn}(s(t))=\left\{\begin{array}{lll}+1, & \text { if } & s(t)>0 \\ 0, & \text { if } & s(t)>0 \\ -1, & \text { if } & s(t)>0 .\end{array}\right.$

\section{B. Radial Basis Function Neural Networks}

We assume $\xi$ of Eq. (11) to the lumped uncertainty and a RBFNNs algorithm with on-line learning rule is proposed in order to determine $\xi$. Figure 2 shows the general structure of RBFFNNs.

The output of RBFNNs can be represented as

$u_{R B F}=\sum_{i=1}^{N} w_{i} O_{i}$.

where $w_{i}$ is the connection weigh factor between the layer 2 and 3. $O_{i}$ is the output of the layer 2 and $u_{R B F}=\xi$.

When the RBF are chosen as Gaussian functions, the output of layer 2 can be represented as [8]

$O_{i}(Z)=\exp \left\{\frac{-\left\|\prod \mathrm{E}-m_{i}\right\|^{2}}{\sigma_{i}^{2}}\right\}$

where $\mathrm{E} \in R^{N}$ is the input vector, $u_{R B F}$ is the output, $w_{i}$ is the synaptic weight, and $m_{i}$ and $\sigma_{i}$ are the mean and the standard deviation of Gaussian functions, respectively.

To derive the on-line learning rule of the means, the standard deviations, and the weights in RBFNNs, the standard gradient descent algorithm is used. First, the energy function $E$ to be minimized is defined as $E=\frac{1}{2}\left(x_{d}-x\right)^{2}=\frac{1}{2} e^{2}$.

In the layer 3 , the partial differential equation to minimize $E$ by the output $\xi$ can be represented as

$L^{3}=-\frac{\partial E}{\partial u_{R B F}}=-\frac{\partial E}{\partial e} \frac{\partial e}{\partial x_{1}} \frac{\partial x_{1}}{\partial u_{R B F}}$

where $L^{3}$ denotes the layer 3 with the output layer. The update of $w_{i}$ is

$\Delta w_{i}=-\eta_{w} \frac{\partial E}{\partial w_{i}}=-\eta_{w} \frac{\partial E}{\partial u_{R B F}} \frac{\partial u_{R B F}}{\partial w_{i}}=\eta_{w} L^{3} O_{i}$

where $\eta_{w}$ is the learning rate.

In the layer 2, the approximated error term needs to be propagated for calculating the error term of the layer 2 as follows:

$L^{2}=-\frac{\partial E}{\partial O_{i}}=-\frac{\partial E}{\partial u_{R B F}} \frac{\partial u_{R B F}}{\partial O_{i}}$

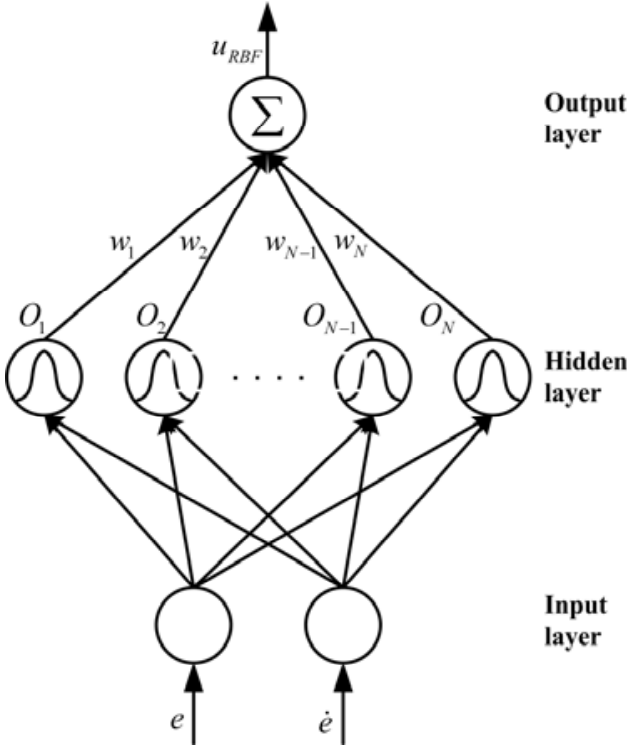

Fig. 2. A general structure of RBFNNs.

and then the mean, and standard deviation of the hidden layer can be updated by using the following equations:

$$
\begin{aligned}
& \Delta m_{i}=-\eta_{m} \frac{\partial E}{\partial m_{i}}=\eta_{m} L^{2} \frac{2\left(x_{i}^{2}-m_{i}\right)}{\left(\sigma_{i}\right)^{2}} \\
& \Delta \sigma_{i}=-\eta_{\sigma} \frac{\partial E}{\partial \sigma_{i}}=\eta_{\sigma} L^{2} \frac{2\left(x_{i}^{2}-m_{i}\right)^{2}}{\left(\sigma_{i}\right)^{3}}
\end{aligned}
$$

where $\eta_{m}$ and $\eta_{\sigma}$ are the learning rates for the mean and standard deviation of the Gaussian function, respectively. Finally, every connect weights between the layers 1 and 2 are defined to unity.

\section{COMPUTER SIMULATION}

In order to evaluate the validity of the proposed control scheme for EHA position control systems, a step input and a sinusoidal reference input were considered as follows:

$x_{d}=2[\mathrm{~cm}]$

$x_{d}=10 \times \sin (\pi t)[\mathrm{cm}]$

Table 1 shows the system parameters of the EHA system which are used to computer simulation.

Figure 3 shows the position errors of the SMC with and without RBFNNs for the step reference input. From Fig. 3, the RMS(root mean square) error of the SMC with RBFNNs is $1.1 \times 10^{-3}$ and it of the SMC without RBFNNs is $2.6 \times 10^{-2}$. In addition, when the external disturbance is excited in 1.5 seconds, the result of the SMC with RBFNNs compared with it of the SMC without 
RBFNNs converges to zero. Figure 4 shows the speed of the motor as the control input depending on the result of Fig. 3.

Figure 5 shows the position errors of the SMC with and without RBFNNs for the sinusoidal reference input. In the case of this reference input, the external disturbance does not exist. Figure 6 shows the speed of the motor as the control input for the sinusoidal reference input, which depends on the result of Fig. 5. From Fig. 5, the RMS error of the SMC with the RBFNNs is $1.2 \times 10^{-3}$ and it of the SMC without RBFNNs is $4.7 \times 10^{-4}$.

These results show that the proposed position control scheme has the desired robustness to system uncertainties.

\section{CONCLUSION}

A robust position control of EHA systems was proposed by using the SMC scheme as the main controller and the RBFNNs as the auxiliary compensator. To verify the robustness and the tracking performance of proposed control scheme, the external disturbance input for a step and a sinusoidal reference inputs are applied to the a computer simulation. From the computer simulation, it was found that the SMC systems with RBFNNs can yield the desired tracking performance and the robustness to the external disturbance.

Table 1 System parameters of the EHA system

\begin{tabular}{c|l|c}
\hline Notation & \multicolumn{1}{|c}{ Description } & Unit \\
\hline$V_{0}$ & $\begin{array}{l}\text { Initial volume of the } \\
\text { chamber }\end{array}$ & $3.712 \times 10^{-4} \mathrm{~m}^{3}$ \\
\hline$A$ & $\begin{array}{l}\text { Pressure area of the } \\
\text { piston }\end{array}$ & $5.58 \times 10^{-3} \mathrm{~m}^{2}$ \\
\hline$M$ & Piston mass & $1.7 \times 10^{3} \mathrm{MPa}$ \\
\hline$\beta_{e}$ & $\begin{array}{l}\text { Effective bulk mod- } \\
\text { ulus }\end{array}$ & $\begin{array}{l}\text { Leakage factor of the } \\
\text { pump }\end{array}$ \\
\hline$L_{f}$ & $\begin{array}{l}\text { Maximum speed of } \\
\text { the motor }\end{array}$ & $178 \mathrm{rad} / \mathrm{s}$ \\
\hline$\omega_{p \max }$ & $\begin{array}{l}\text { Volumetric capacity } \\
\text { of the pump }\end{array}$ & $1.591 \times 10^{-6} \mathrm{~m}^{3} / \mathrm{Pad}$ \\
\hline$C_{p}$
\end{tabular}

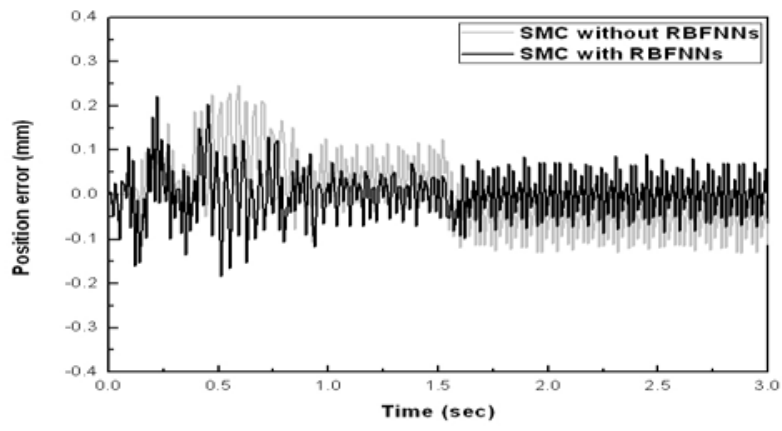

Fig. 3 Position errors of the SMC with and without RBFNNs for the step reference input

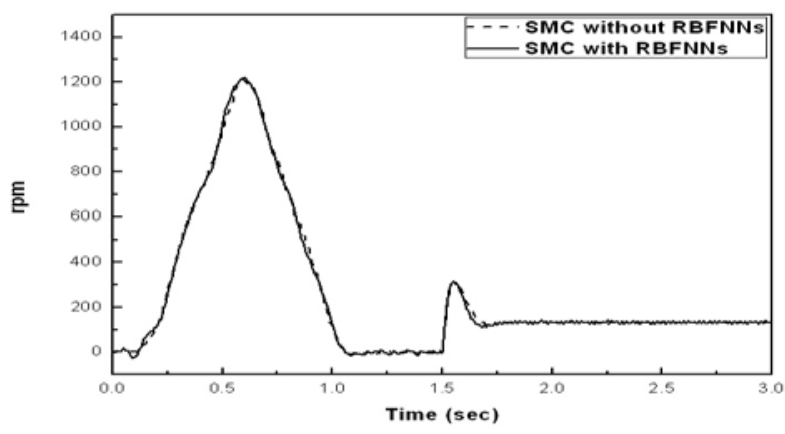

Fig. 4 Speed of the motor as the control input for the step reference input

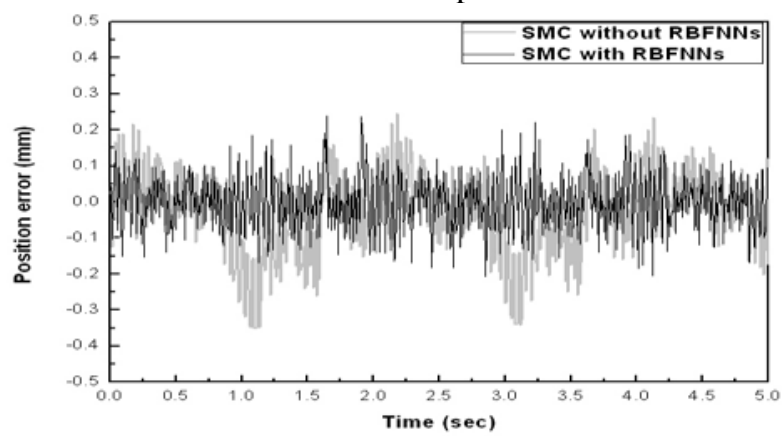

Fig. 5 Position errors of the SMC with and without RBFNNs for the sinusoidal reference input

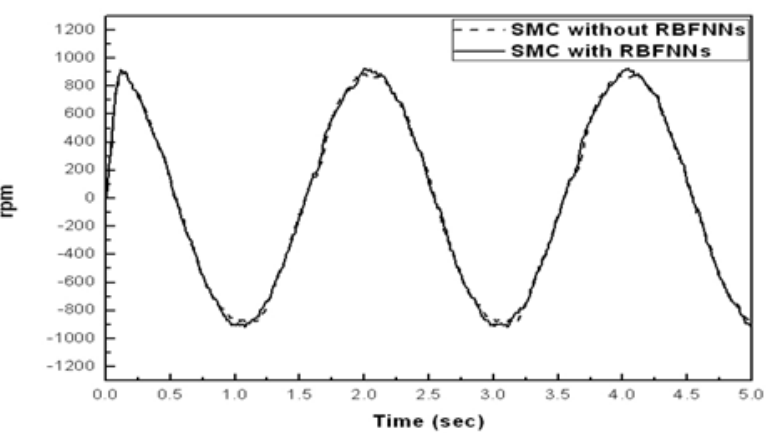

Fig. 6 Speed of the motor as the control input for the sinusoidal reference input 


\section{ACKNOLEGEMENT}

This work was supported by the Technology Innovation Program funded by the Ministry of Knowledge Economy(MKE, Korea)

\section{REFERENCES}

[1] Navarro, R., "Performance of an Electro-Hydrostatic Actuator on the F-18 Systems Research Aircraft”, Dryden Flight Research Center, Edwards, California, USA, 1997

[2] Habibi, S. and Goldenberg, A., Design of a new highperformance electro-hydraulic actuator, IEEE Trans. Mechatronics, 5(2), pp. 158-164. 2000.

[3] Zhou, K. and Doyle, J., Essentials of Robust Control, Prentice Hall, New Jersey, USA, 1998.

[4] Kristic, M., Kanellakopoulos, I., and Kokotovic, P., Nonlinear and Adaptive Control Design, Wiley Interscience, New York, USA, 1995.

[5] Edwards, C. and Spurgeon, S., Sliding Mode Control; Theory and Applications, Taylor \& Francis, Padstow, UK, 1998.

[6] Merritt, H. E., Hydrostatic control systems, Wiley, New York, USA, 1967.

[7] Kim, H. M., Park, S. H., Lee, J. M., and Kim, J. S., “A Robust Control of Electro Hydrostatic Actuator Using the Adaptive Back-Stepping Scheme and Fuzzy Neural Networks”, Vol. 11(2), pp. 227-236, 2010.

[8] Lin, F. J., Shen, P. H., Yang, S. L., and Chou, P. H., "Recurrent Radial Basis Function Network-based Fuzzy Neural Network Control for Permanent Magnet Linear Synchronous Motor Servo Drive”, IEEE Transactions on magnetic, Vol. 42(11), pp. 3694-3750, 2006. 